DOI: $10.33581 / 2020-20-41-50$

\title{
ЛУЧШИЕ ПРАКТИКИ ОБУЧЕНИЯ РАБОТЕ С ДАННЫМИ: ОПЫТ ЗАРУБЕЖНЫХ УНИВЕРСИТЕТСКИХ БИБЛИОТЕК И ЦЕНТРОВ
}

\section{BEST PRACTICES FOR DATA MANAGEMENT LEARNING: FOREIGN UNIVERSITY LIBRARIES' AND PROFESSIONAL RESEARCH CENTRES' EXPERIENCE}

Маслова Анастасия Васильевна - заведуюший отделом поддержки исследований Научной библиотеки РАНХиГС (Россия), maslovaav@ranepa.ru

Maslova A. V. - Head of the Research Support Department of the RANEPA Library(Russian Federation), maslova-av@ ranepa.ru

Аннотация: на сегодняшний день не осталось сомнений в том, что в исследовательском сообществе растет потребность в работе с данными. Однако, помимо самих данных, исследователям нужна дополнительная поддержка, ведь прочесс планирования, сбора, обмена, хранения данных также требует усилий и средств. Сейчас существует несколько международных иентров и библиотечных инициатив, которые помогают исследователям организовать правильный процесс работы с данными. Многие из них выходят на крупные образовательные площадки и делятся своим опытом. В данном тексте рассмотрим лучшие практики и инициативы по обучению работы с данными, которые предлагают ведущие исследовательские центры и университеты, профессиональные онлайн курсы по менеджменту данных в академической среде.

Abstract: No doubt, that there is a growing need in the research community to work with data. In addition to the data itself, researchers need support, because the process of planning, collecting, sharing, and storing data also requires effort. Now there are several international centers and library initiatives that help researchers organize the correct process of working with data. Many of them use 
large educational platforms and share their experience. We will look at the best practices and initiatives for training in working with data offered by leading research centers and universities, professional online courses on data management.

Ключевые слова: менеджмент данных, исследовательские данные, онлайн курсы по менеджменту данных, обучение работе с данными.

Keywords: Data Management, Research Data, professional online courses on data management, data curation.

Темы, касающиеся использования исследовательских данных, сегодня широко обсуждаются разными специалистами в академической среде. Несмотря на фундаментальную важность обучения тому, как именно необходимо управлять данными (хранить, передавать или искать), в подобных дискуссиях основной упор делается скорее на обсуждение инструментов для анализа данных, например, возможности их обработки c помощью Python или R. B этом тексте речь пойдет не об инструментах, а как раз о практиках и инициативах по обучению работе с данными, которые предлагают ведущие исследовательские центры, университеты и профессиональные онлайн курсы по менеджменту данных.

Прежде чем начать разговор о конкретных практиках обучения работе с данными необходимо пояснить, почему важно говорить о менеджменте данных именно сейчас, и почему в рамках разговора об этом желательно делать акцент не на технологиях и инструментах анализа данных, а именно на обучении людей и закреплении у них навыков работы с исследовательскими данными.

Менеджмент данных - это целый набор разных практик, направленных на хранение, поиск, распространение и обмен данными. Помимо этого, деятельность по управлению данными также включает в себя планирование, 
форматирование,

документирование,

анонимизацию

данных

и управление доступом к данным. Озвученные практики, несмотря на рутинность, в действительности требуют, как минимум, большего вовлечения и концентрации со стороны исследователей, библиотекарей или центров по поддержке исследований, чем просто изучение отдельных программ и технологий. Простое понимание универсальных методов обработки данных, например, с помощью программирования, в отличии от полноценного обучения навыкам поиска, распространения и хранения данных, не дает исследователям комплексного понимания того, куда в настоящий момент движется та или иная научная дисциплина. К тому же, когда исследователь четко понимает, какие исследовательские данные ему нужны, где их можно найти, как правильно их хранить, как описывать, как должен быть спланирован процесс использования и распространения данных, это дает возможность не просто правильно использовать данные в исследованиях, но и делать их более качественно.

Акцент на обучении работе с данными необходим сегодня по нескольким причинам. В долгосрочной перспективе не технологии, а именно навыки качественного управления данными позволят, во-первых, поддерживать коммуникацию между отечественными и зарубежными исследовательскими коллективами, а во-вторых, сохранять данные для будущих исследователей. Также в краткосрочной перспективе не высококлассные навыки программирования, a именно грамотно выстроенный менеджмент данных поможет ученым лучше продвигать результаты своих научных исследований и успешно сотрудничать с авторитетными мировыми научными журналами, издательствами, фондами, грантодателями, научно-исследовательскими институтами.

На сегодняшний день курсы по менеджменту данных уже не редкость. Существуют зарубежные центры, которые занимаются темой обучения работе с данными, а также профессиональные онлайн курсы. Некоторые 
из них представлены на популярных образовательных платформах, вроде Coursera, Canvas, FutureLearn. Готовые обучающие курсы по менеджменту данных направлены на развитие конкретных исследовательских и административных навыков у разных целевых аудиторий. Исследователи, благодаря таким курсам, смогут разобраться, как управлять «своими» данными и работать с «готовыми». Для преподавателей и студентов курс по управлению данными может стать хорошим подспорьем для того, чтобы лучше разобраться в изучаемой научной дисциплине. Для научных администраторов и библиотекарей опыт зарубежных центров по управлению данными поможет лучше понять, как данные встраиваются в научные исследования. Такой курс также поможет тем, кто вовлечен в процесс исследования только с административной стороны, освоить новые формы поддержки исследований в организации и создать эффективную организационную культуру.

В данном тексте рассмотрим три ярких примера онлайн курсов от зарубежных библиотек и центров, которые посвящены обучению работе с данными.

Первый онлайн курс называется «Delivering Research Data Management Services». Данный курс разрабатывался Эдинбургским университетом, известным в сфере научной информации центом «Digital Curation Centre» и Центром научных исследований Нидерландов. Целью курса является обучение современных библиотекарей и научных администраторов принципам менеджмента данных для того, чтобы в дальнейшем они могли организовать правильные структуры по поддержке исследований в своих университетах или иных организациях.

Блоки этого курса состоят из небольших статей и коротких видео, основной объем составляют задания, которые подразумевают развернутые ответы и возможность участия в дискуссии в чате по обозначенной теме. Авторы курса считают, что таким образом участники кроме теории могут 
получить больше практических советов, полезных примеров того, как можно использовать и внедрять инициативы по управлению данными в стратегию своей организации. Однако, помимо стратегии и планирования, особенно ценным кажется и то, что авторы курса предлагают уже готовые советы по разработке тренингов по работе с данными для исследовательских коллективов и организации в целом.

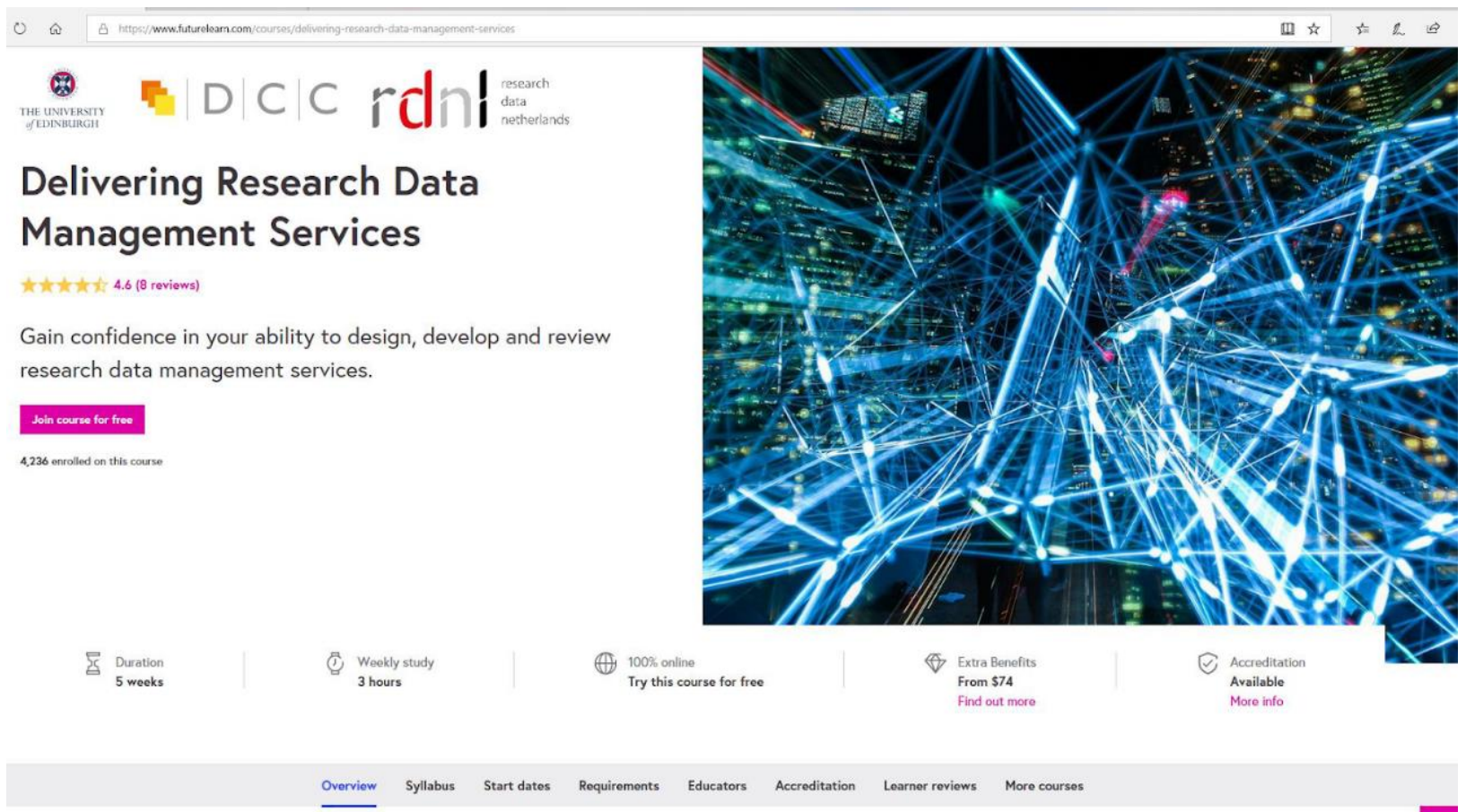

Pис. 1. Главная страница курса «Delivering Research Data Management Services» на платформе FutureLearn

Следующий курс «Research Data Management Librarian Academy (RDMLA)» также нацелен на профессиональное обучение библиотекарей, научных администраторов и других специалистов по информации навыкам управления данными. Разработчики курса - библиотекари, которые уже работают с данными в центрах поддержки исследований и готовы поделиться своим опытом. Программа данного курса, как и в предыдущем случае, фокусируется на необходимых знаниях в области менеджмента данных.

Курс состоит из восьми самостоятельных учебных программ, которые охватывают основы лицензирования исследовательских данных, советы 
и рекомендации по практике внедрения методов работы с данными в библиотеках и обзор инструментов для анализа данных. В отличии от предыдущего онлайн курса, в рамках данной программы в качестве заданий выступают проверочные тесты в конце блока или видеоурока. Для успешного прохождения онлайн курса не требуется слушать все восемь предложенных программ. В целом курс не предполагает дискуссии и обмена опытом между участниками и подходит тем, кому удобнее самостоятельно планировать свое обучение, выбирая именно те части, которые кажутся наиболее полезными.

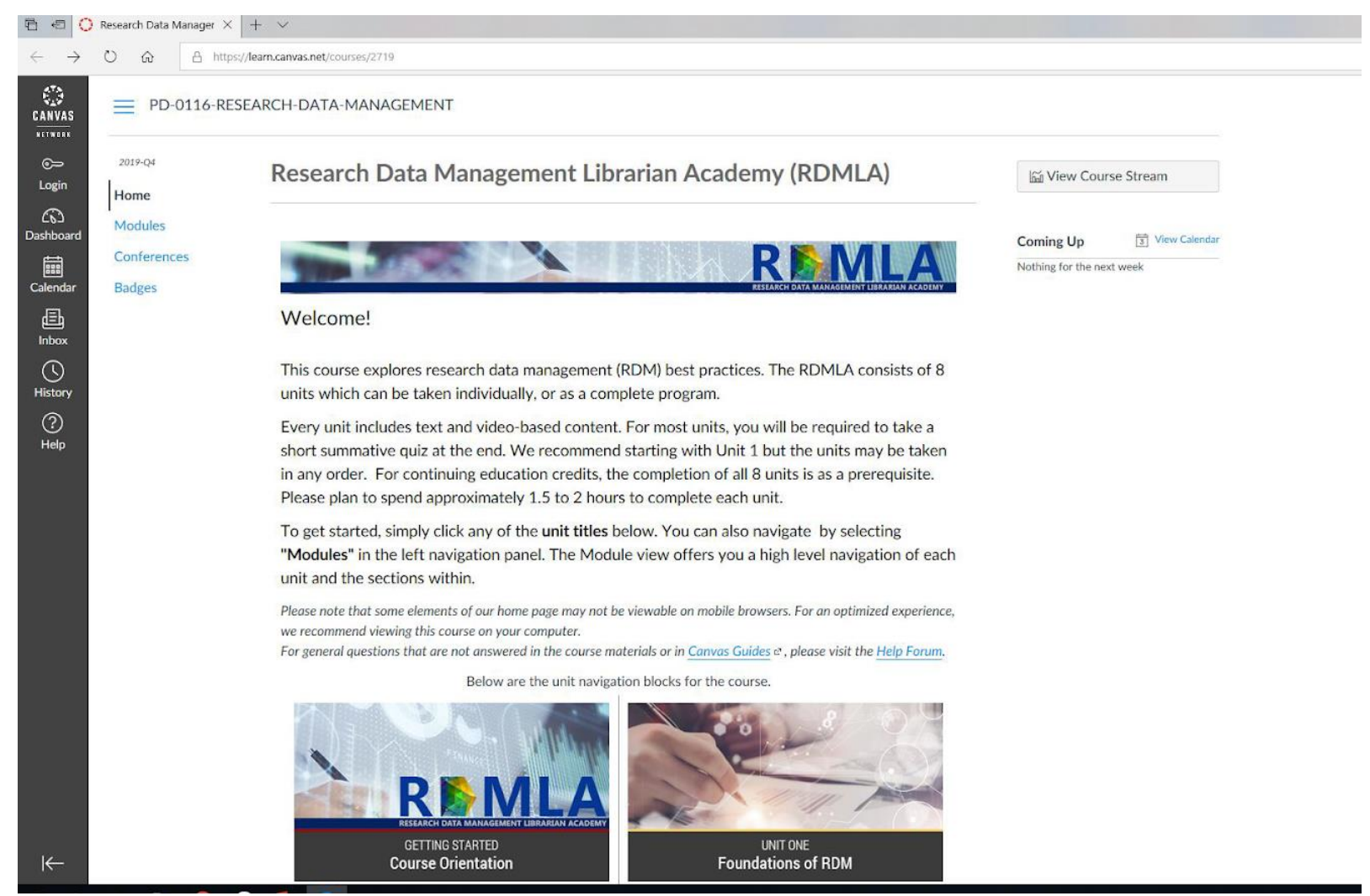

Puc. 2. Главная страница курса «Research Data Management Librarian Academy (RDMLA)» на платформе Canvas

Третий онлайн курс, который будет рассматриваться в данном тексте, называется «Research Data Management and Sharing». В отличии от предыдущих, этот курс предназначен для более широкой аудитории и может быть полезен как исследователям, студентам и преподавателям, так библиотекарям и научным администраторам. Сам курс нацелен не столько на развитие конкретных навыков управления данными, сколько 
на качественное объяснение того, что из себя представляют исследовательские данные и как выглядит цикл создания и использования данных. Помимо общей стратегической части, в рамках курса делается упор на объяснение принципов файлового менеджмента для тех, кто создает данные или пользуется готовыми данными из разных областей.

Подготовкой материалов для данного онлайн курса занимались ведущие центры поддержки данных, а также библиотеки Эдинбургского университета и университета Северной Каролины. Несмотря на то, что курс отличается от остальных по наполнению и целевой аудитории, структура учебной программы у всех курсов похожа. Занятия также проходят в формате видео, к каждому тематическому блоку прилагаются статьи и полезные ссылки, задания предполагают развернутый ответ по темам курса или тест. Письменные задания можно обсудить с другими слушателями и получить фидбэк. Такая практика обмена мнениями не просто приветствуется, но и порой предполагается организаторами курса как обязательная.

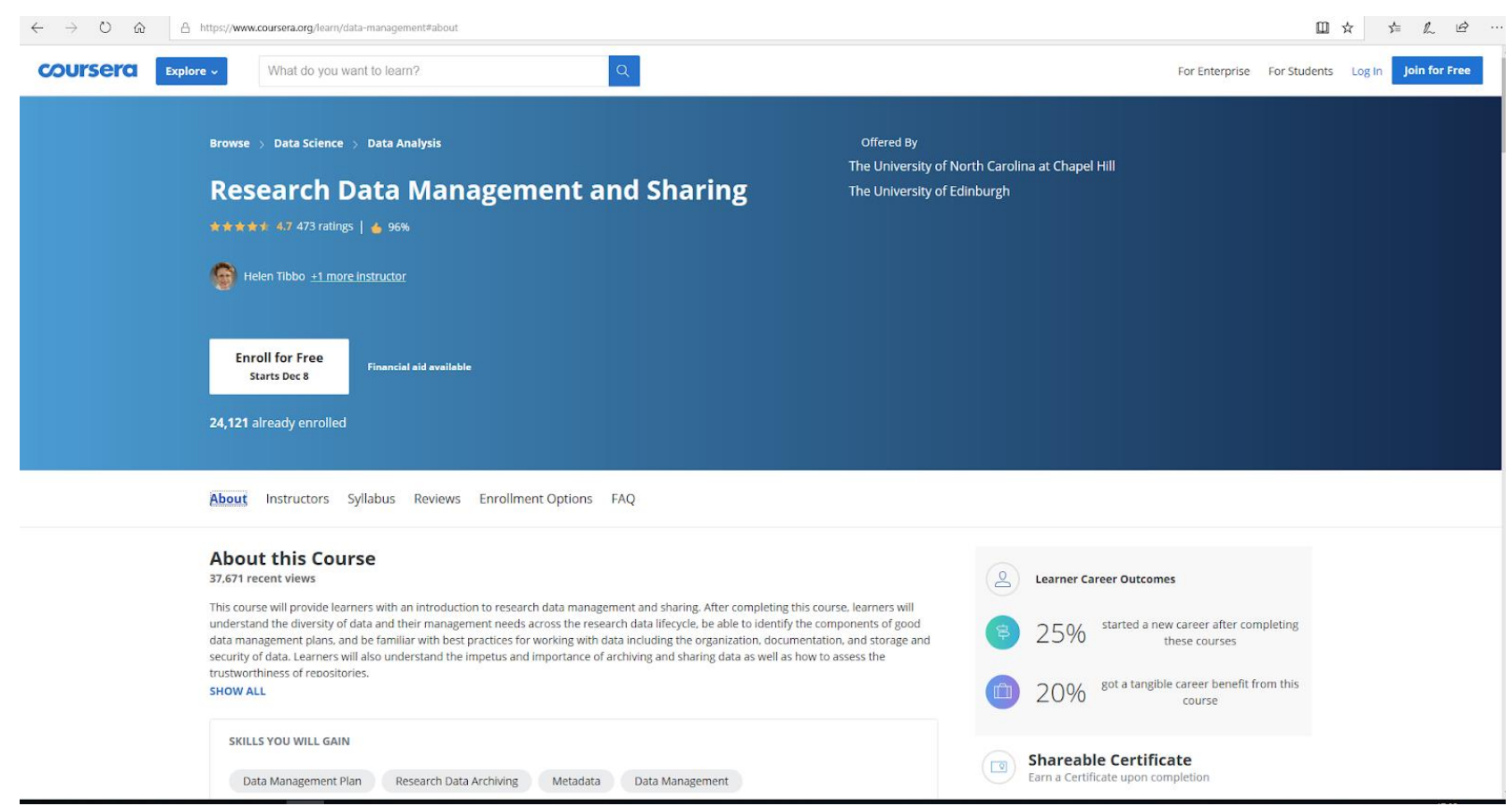

Pис. 3. Главная страница курса «Research Data Management and Sharing» на платформе Coursera 
Основные темы трех затронутых курсов вращаются вокруг одних и тех же областей, которые можно назвать фундаментальными в вопросах управления исследовательскими данными. Во-первых, во всех курсах упоминается цикличность процесса создания и использования данных. Традиционно авторы курсов выделяют, как минимум, пять этапов работы с данными: поиск, планирование, описание, архивирование и публикация. Далее в каждом курсе в зависимости от выбранной целевой аудитории детализируется тот или иной цикл работы с данными или способ организации работы с данными. Во-вторых, в каждом из трех курсов обсуждается тема планирования, поскольку создание продуманного и качественного плана является основой работы с данными, как для библиотекарей и научных администраторов, так и для исследователей. В-третьих, в качестве основной темы для рассмотрения вопроса менеджмента данных авторы курсов выбирают файловый менеджмент. На примере правильного наименования, хранения файлов авторы курсов помогают развивать навыки управления данными в рамках организации или даже небольшого исследовательского коллектива.

Однако, помимо готовых знаний по теме качественного управления данными от зарубежных коллег, для тех, кто заинтересован в продвижении темы, например, среди библиотекарей или научных администраторов, также найдется ниша, которую можно не просто занять, но и начать активно развивать. Вместо попыток охватить сразу все тонкости управления данными, изначально можно остановиться на двух простых и понятных пунктах: обучение работе с «готовыми» данными и обучение работе со «своими» данными для исследователей, преподавателей и студентов.

Для того, чтобы привить правильную культуру работы с «готовыми» данными, для начала можно просто подготовить общие встречи или лекции, посвященные плюсам использования вторичных данных в научных работах. Также помимо объяснения, зачем ученые сегодня могут использовать чужие 
данные, можно рассказать о том, где искать «готовые» данные. Для таких целей можно проводить тренинги по поиску в специальных поисковиках, которые аккумулируют данные по профессиональным областям, или в мета поисковиках, которые аккумулируют данных из репозиториев. Например, для исследователей, преподавателей и студентов можно рассказать о возможностях поиска в Mendeley Data или любой другой похожей базе данных. Если есть потребность охватить более конкретные области управления «готовыми» данными, можно подготовить ряд выступлений на тему хранения данных и правильного цитирования чужих данных в исследовательских и учебных работах.

Если в вашей организации есть сотрудники, которые сами генерируют исследовательские данные, то со стороны библиотекарей или научных администраторов не лишним будет проконсультировать их по созданию плана работы с исследовательскими данными, а также по вопросам файлового менеджмента, а именно, правильного именования файлов, созданию дополнительных и основных версий данных, безопасному хранению. Помимо прочего, можно проводить тренинги по стандартам создания метаданных: правильного документирования последовательности работы с данными и выбора необходимого формата описания собранных данных.

Для тех, кто хочет лучше разобраться в теме управления данными, приводим список полезных инструментов и материалов по работе с данными, взятых из учебных онлайн курсов ведущих зарубежных центров и библиотек:

\section{Репозитории данных:}

- $\quad \underline{E A S Y}$ (данные гуманитарных и социальных наук)

- $\quad$ 4TU.Centre for research data (данные технических наук)

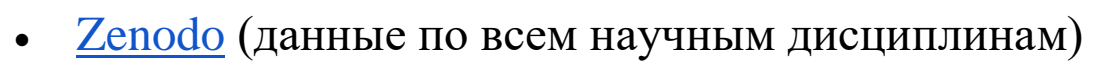

- $\quad$ Dryad (данные по всем научным дисциплинам)

- $\quad$ Mendeley Data (поисковик данных) 


\section{Центры по работе с данными:}

- Digital Curation Centre (DCC)

- Center for Research Libraries

- Research Data Netherlands

- The Research Data Alliance

Академические подразделения и библиотеки, работающие с данными:

- Research Data Service University of Edinburgh

- The TU Delft Library

- Research Data Service University of Glasgow

- LIBER

\section{Полезные инструменты:}

- Data Management Plans online

- MANTRA 